\title{
Comparação das propriedades mecânicas de juntas de alumínio obtidas por soldagem a laser (LBW), por friction stir welding (FSW) e rebitadas para aplicação em estruturas aeronáuticas
}

\section{(Comparing mechanical behavior of aluminum welds produced by Laser Beam Welding (LBW), Friction Stir Welding (FSW) and riveting for aeronautic structures)}

\author{
${ }_{1,}^{1, *}$ Rafael Humberto Mota de Siqueira, ${ }^{2}$ Aline Capella de Oliveira, ${ }^{3}$ Rudimar Riva, ${ }^{3}$ Antonio Jorge Abdalla e ${ }^{3}$ Milton Sérgio Fernandes \\ de Lima \\ ${ }^{1}$ Instituto Tecnológico de Aeronáutica - ITA/DCTA, São José dos Campos, São Paulo, Brasil, rafaelhmota@yahoo.com.br \\ ${ }^{2}$ Instituto de Ciência e Tecnologia - ICT/UNIFESP, São José dos Campos, São Paulo, Brasil \\ ${ }^{3}$ Instituto de Estudos Avançados - IEAv/DCTA, São José dos Campos, São Paulo, Brasil, \\ msflima@gmail.com
}

\section{Resumo}

Três processos de união de perfis de alumínio concorrem para a manufatura de painéis estruturais (união entre revestimento e reforçadores) aeronáuticos: rebitagem, Friction Stir Welding (FSW) e soldagem a laser (Laser Beam Welding - LBW). Esses processos têm vantagens e desvantagens particulares, as quais foram analisadas no presente estudo focando, especificamente, nas propriedades de tração do conjunto unido. Dois ensaios mecânicos foram considerados, T-pull test e Hoop test. No que tange ao ensaio T-pull, as amostras soldadas por LBW apresentam maior capacidade de suportar cargas. Isso se deve à melhor distribuição de cargas, quando a solicitação mecânica se faz pelo reforçador. No caso do ensaio Hoop, onde a solicitação mecânica se dá exclusivamente no revestimento, os resultados obtidos por FSW são notadamente superiores aos obtidos pelos demais processos, em termos de tensão máxima, tensão de escoamento e de alongamento máximo. Conclui-se que tanto o LBW quanto o FSW são métodos de soldagem com possibilidade de substituir a rebitagem comumente aplicada nas aeronaves comerciais.

Palavras-chave: Soldagem a laser (LBW); Friction Stir Welding (FSW); Rebitagem.

Abstract: Three welding processes for aluminum parts have been considered for the aircraft fabrication: riveting, friction stir welding (FSW) and laser beam welding (LBW). These processes have advantages and threats, which were analyzed in the present work focusing on T-pull and Hoop tensile properties. Concerning T-pull tests, LBW coupons presented higher ability to withstand the applied loads. This was due to the better distribution of loads when the strain is done to the stringer direction. In the case of the Hoop tests, which stress only the skin, the results obtained after FSW were notably higher in terms of ultimate tensile stress, yield stress and maximum strain. It was concluded that both LBW and FSW could replace riveting usually applied for commercial aircraft manufacturing.

Keywords: Laser Beam Welding (LBW); Friction Stir Welding (FSW); Riveting.

\section{Introdução}

$\mathrm{Na}$ indústia aeronáutica existe uma busca constante pela redução de peso nas aeronaves, compreendendo a utilização de motores menores e mais potentes, reduzindo o consumo de combustível e, portanto, diminuindo a emissão de gases nocivos na alta atmosfera [1].

$\mathrm{Na}$ estrutura de uma aeronave existem duas formas de redução de peso. A primeira é a utilização de materiais leves, tais como ligas de alumínio e materiais compósitos. A segunda maneira é a utilização de técnicas de união que não utilizem um material adicional, como rebites ou da sobreposição de chapas.

Recebido em 17/09/2013, texto final em05/05/2014.

DOI: 10.1590/0104-9224/SI1902.06
O setor aeronáutico mundial, desde o final do século passado até os dias atuais, vem estudando processos alternativos de junção em estruturas aeronáuticas, em substituição ao processo tradicional de rebitagem [2].

Neste sentido, os processos avançados de soldagem são alternativas bastante promissoras na substituição do processo de rebitagem. Atualmente, técnicas de soldagem a laser (Laser Beam Welding - LBW) e Friction Stir Welding (FSW) já estão sendo utilizados na indústria aeronáutica $[2,3]$.

Empresas como a Airbus estão soldando a laser os painéis laterais e inferiores de aviões da classe 300, ao invés de rebitálos. A fábrica da Airbus em Toulouse dispõe de várias estações de trabalho com lasers para soldar os reforçadores com o revestimento.

A soldagem a laser é capaz de substituir o processo de rebitagem, sendo utilizada para soldagem de juntas do tipo $\mathrm{T}$,

* Autor para quem toda a correspondência deve ser encaminhada. 
ou seja, entre a fuselagem e reforçador. A eliminação das abas de rebitagem dos reforçadores e dos materiais de preenchimento entre o reforçador e o revestimento podem levar a uma redução de $5 \%$ no peso dos componentes estruturais da aeronave. Com o desenvolvimento de novos materiais, mais leves e resistentes e com o avanço da tecnologia de soldagem a laser, os especialistas da área estimam que a redução no peso total das aeronaves pode chegar a $15 \%$. [4].

Mais recentemente, o processo FSW foi qualificado para a produção de juntas de topo (butt joints) no fechamento do barril (segmento de fuselagem) [5].

O sucesso da aplicação da soldagem nas aeronaves de grande porte da série A3XX motivou recentemente a implementação de um grande projeto de pesquisa europeu, denominado Clean Sky Joint Technology Initiative para estudar a viabilidade desta tecnologia em aeronaves de menor porte, utilizadas na aviação regional. No projeto, procura-se demonstrar que os ganhos em redução de peso e custo de fabricação podem ser estendidos para este tipo de aeronave, utilizando novas ligas de alumínio-cobrelítio [1].

No conhecimento dos autores, dados da literatura não exibem uma comparação direta entre os métodos de junção por rebites, FSW e LBW por meio de ensaios mecânicos nos conjuntos reforçador-revestimento (stringer-skin) de alumínio de alta resistência.

Este trabalho propõe a comparação entre juntas soldadas a laser e por friction stir welding com o tradicional processo de união com rebites. Para esta finalidade foram realizados ensaios de tração, T-pull test e Hoop test, com análises macroscópicas subsequentes da superfície de fratura dos corpos-de-prova (CDPs) submetidos aos ensaios.

\section{Materiais e Métodos}

Os corpos-de-prova rebitados e soldados por FSW foram fornecidos pela Empresa Brasileira de Aeronáutica S.A. (EMBRAER). Os corpos-de-prova seguiram as normas da EMBRAER, com os mesmos parâmetros de processo utilizados na confecção da fuselagem das aeronaves por ela produzidas.

Os CDPs rebitados, Figura 1 (a), foram fabricados, sem selante, utilizando como revestimento uma chapa com $130 \mathrm{~mm}$ de comprimento, $44 \mathrm{~mm}$ de largura e espessura de $1,3 \mathrm{~mm}$. O reforçador com formato de cantoneira com abas de $25 \mathrm{~mm}$ de largura, comprimento de $44 \mathrm{~mm}$ e espessura de $1,3 \mathrm{~mm}$, foi fixado no revestimento utilizando-se dois rebites de diâmetro de $4,0 \mathrm{~mm}$, separados por uma distancia de $25 \mathrm{~mm}$. Os rebites atendem à norma militar MS14218AD4-4.

Para a confecção dos corpos-de-prova por FSW, os seguintes parâmetros foram utilizados: velocidade de avanço da ferramenta de $700 \mathrm{~mm} / \mathrm{min}$, velocidade de rotação de $1700 \mathrm{RPM}$ e força normal de $4700 \mathrm{~N}$. A ferramenta utilizada no equipamento de FSW possui um pino não-retrátil de $8 \mathrm{~mm}$ de largura com ombro de $15 \mathrm{~mm}$ de diâmetro. A Figura 1 (b) mostra uma imagem do CDP fabricado por FSW em que foi utilizada uma chapa com $130 \mathrm{~mm}$ de comprimento, $45 \mathrm{~mm}$ de largura e espessura de 1,3 $\mathrm{mm}$ como material do revestimento. $\mathrm{O}$ reforçador, também em
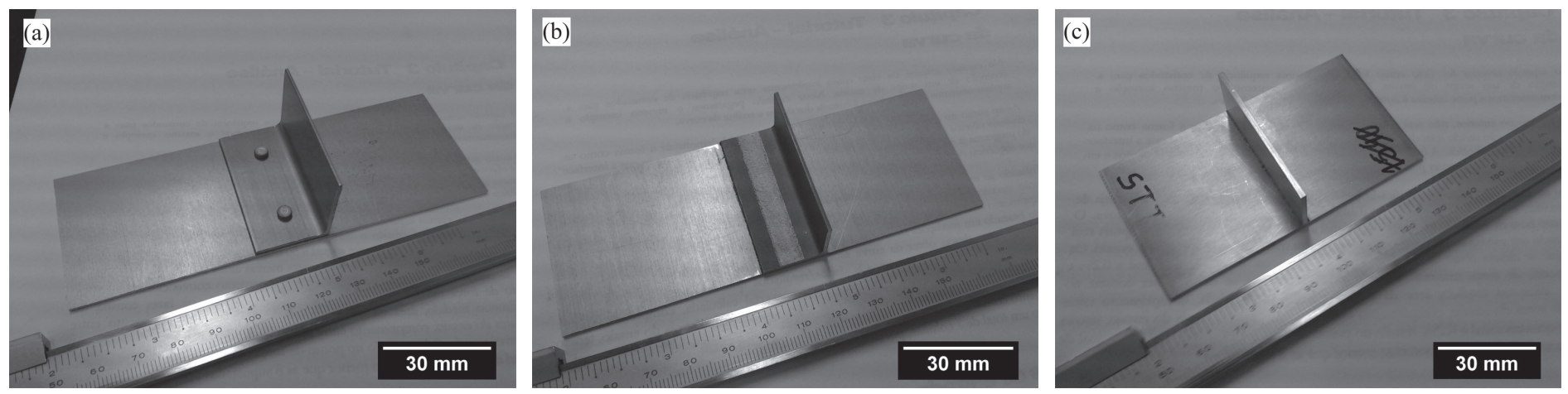

Figura 1. Imagens dos três corpos-de-prova utilizados: (a) Rebitado, (b) FSW e (c) LBW.

Tabela 1. Ligas de alumínio, utilizadas, nos diferentes processos de fabricação.

\begin{tabular}{|l|c|c|c|}
\hline Processo & Revestimento & Reforçador & Rebite \\
\hline Rebitagem & AA2024 & AA7050 & AA2117 \\
\hline FSW & AA2024 & AA7050 & - \\
\hline LBW & AA6013 & AA6013 & - \\
\hline
\end{tabular}

Tabela 2. Propriedades mecânicas das ligas de alumínio utilizadas na confecção dos CDPs $[9,10]$.

\begin{tabular}{|l|c|c|c|c|}
\hline & AA7050-T7651 & AA2024 -T3 & AA2117-T4 & AA6013-T4 \\
\hline Tensão Máxima (MPa) & 552 & 483 & 296 & 350 \\
\hline Tensão de Escoamento (MPa) & 490 & 345 & 165 & 230 \\
\hline Alongamento Máximo (\%) & 11 & 10 & 27 & 28 \\
\hline
\end{tabular}


formato de cantoneira com abas de $25 \mathrm{~mm}$, tem um comprimento de $44 \mathrm{~mm}$ e espessura de $1,3 \mathrm{~mm}$.

Para o processo de soldagem a laser foi utilizado um laser de Yb-fibra (IPG modelo YLR-2000) com 2,0 kW de potência média e qualidade de feixe $\left(\mathrm{M}^{2}\right)$ de aproximadamente 9 e distribuição de intensidade aproximadamente gaussiana $\left(\mathrm{TEM}_{00}\right)$. Os parâmetros de soldagem utilizados foram: potência média de $1,5 \mathrm{~kW}$, ângulo de soldagem de $12,5^{\circ}$, velocidade de soldagem de $6,0 \mathrm{~m} / \mathrm{min}$ e hélio como gás de processo com fluxo de $40 \mathrm{~L} / \mathrm{min}$. Os parâmetros de soldagem autógena foram definidos em estudos realizados por Oliveira e Siqueira [6,7].

Os CDPs soldados a laser, Figura 1 (c), foram fabricados utilizando como revestimento uma chapa com $100 \mathrm{~mm}$ de comprimento, $44 \mathrm{~mm}$ de largura e espessura de 1,6 $\mathrm{mm}$. Já o reforçador apresenta um comprimento de $44 \mathrm{~mm}$ de comprimento, $20 \mathrm{~mm}$ de largura e espessura de 1,6 $\mathrm{mm}$.

Os corpos-de-prova foram confeccionados em diferentes ligas de alumínio, pois os processos de união por rebites e por FSW, realizados pela indústria aeronáutica nacional, utilizam as ligas discriminadas na Tabela 1. A liga AA 6013 foi selecionada para a confecção dos CDPs soldados a laser, por ter sido utilizada na fabricação das aeronaves da AIRBUS. Adicionalmente, a combinação das ligas AA2024 e AA7050 utilizadas no processo de união por FSW tem um baixo grau de soldabilidade, por laser. Na Tabela 2 são apresentadas as propriedades mecânicas das ligas de alumínio AA7050, AA2024, AA2117 e AA6013.

$\mathrm{Na}$ Figura 2, é apresentado um gráfico que relaciona resistência mecânica e ductilidade para as quatro ligas utilizadas na confecção dos CDPs. Observa-se que as ligas de maior resistência, AA 7050 e AA 2024, possuem os menores valores de ductilidade.

Os ensaios de tração foram realizados em uma máquina universal de ensaios (EMIC modelo DL com $100 \mathrm{kN}$ de capacidade), com uma velocidade de ensaio de $1,0 \mathrm{~mm} / \mathrm{min}$ em temperatura ambiente. Foram realizados ensaios de tração T-pull e Hoop, com o objetivo de analisar as propriedades mecânicas do reforçador e do revestimento, respectivamente. Para cada tipo de junta, três corpos-de-prova foram ensaiados.

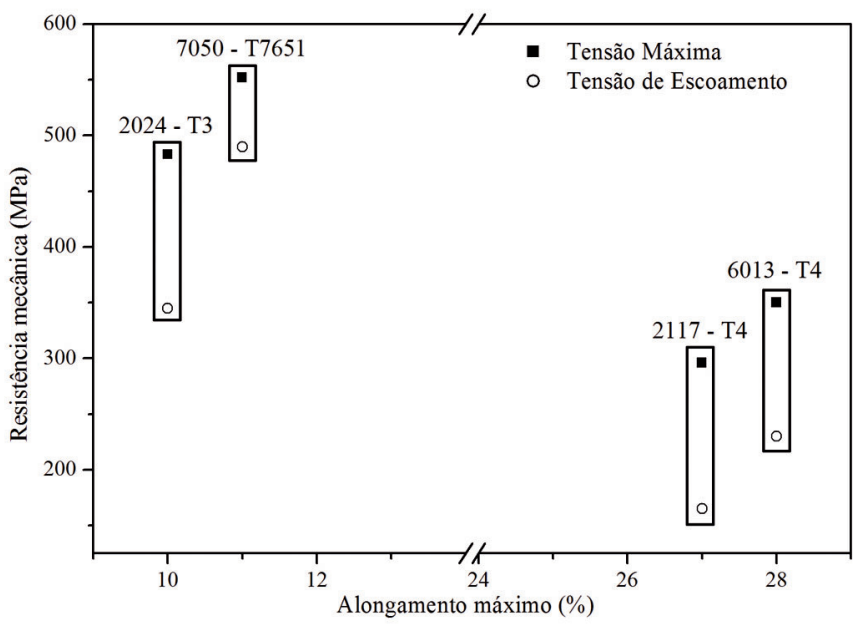

Figura 2. Gráfico da resistência mecânica versus alongamento máximo para os materiais de base.

O ensaio Hoop consiste em tracionar uniaxialmente o revestimento com um reforçador soldado ou rebitado ao centro, sendo que o reforçador está situado perpendicularmente à tensão aplicada. O ensaio de tração T-pull é realizado fixandose firmemente o revestimento sobre uma base e tracionando o reforçador, transferindo o esforço mecânico à região da união. Para o ensaio T-pull foi necessário à utilização de um suporte, onde o corpo-de-prova foi acomodado e apenas o reforçador foi solicitado, como mostrado na Figura 3 [8]. Devido a geometria dos corpos-de-prova do ensaio de tração T-pull utilizou-se o valor de deslocamento e não de deformação para comparar as três técnicas de junção.

\section{Resultados e discussões}

A Figura 4 mostra o gráfico da carga versus deslocamento para o ensaio de tração T-Pull para os três processos de junção. Os valores de cargas e deslocamentos máximos obtidos nesses ensaios são apresentados na Tabela 3. Comparando os limites

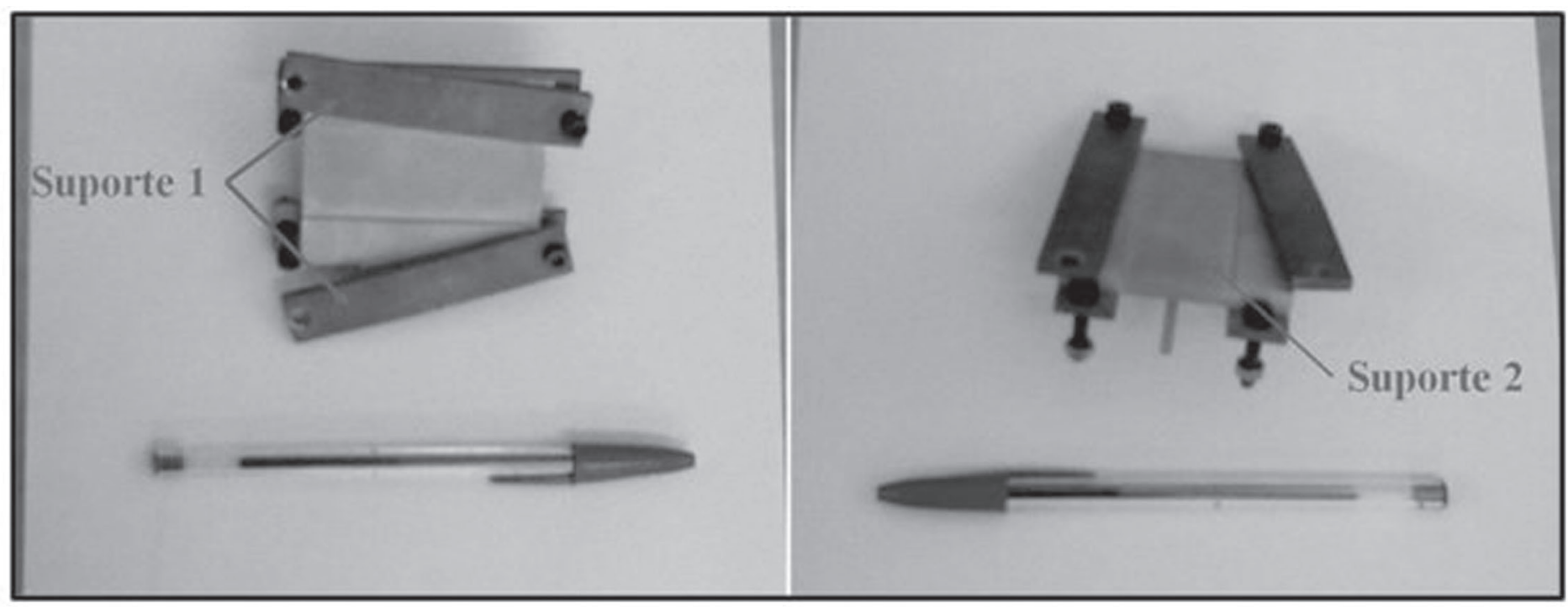

Figura 3. Dispositivo utilizado para a fixação dos CDPs no ensaio de tração $T-P u l l$. 
de resistência das amostras nas três condições propostas, observa-se que as amostras soldadas a laser apresentaram uma capacidade para suportar cargas $133 \%$ maior em relação às amostras rebitadas. As amostras soldadas por FSW revelaram uma capacidade para suportar cargas um pouco menor, 91\% da capacidade apresentada pelas amostras rebitadas. Já os deslocamentos, medidos no eixo da máquina de tração, nos três processos de junção, apresentaram valores similares. No presente estudo, a capacidade de suportar carga da junta foi considerada como o valor máximo de carga na qual a junta resistiu sem romper.

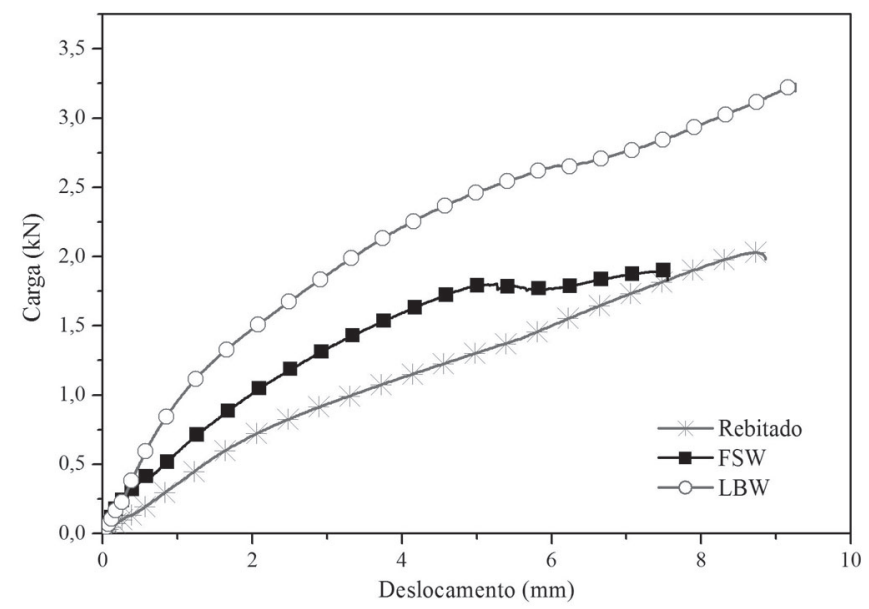

Figura 4. Gráfico carga versus deformação para os três corposde-prova considerados.
Tabela 3. Valores das cargas e dos deslocamentos máximos para as juntas no ensaio de tração T-Pull.

\begin{tabular}{|l|c|c|}
\hline Técnica & Carga Máxima $(\mathrm{kN})$ & $\begin{array}{c}\text { Deslocamento } \\
\text { Máximo }(\mathrm{mm})\end{array}$ \\
\hline FSW & $2,0 \pm 0,7$ & $8 \pm 1$ \\
\hline LBW & $2,9 \pm 0,5$ & $8 \pm 2$ \\
\hline Rebitado & $2,2 \pm 0,2$ & $9 \pm 1$ \\
\hline
\end{tabular}

Para o ensaio de tração T-Pull foi considerado carga (força) e deslocamento ao invés de tensão e deformação, pois, conforme mostrado na Figura 5, os CDPs passam por dois estágios de deformação mecânica. Para os CDPs rebitados, Figura 5 (a), e soldados por FSW, Figura 5 (b), em um primeiro momento existe uma força cisalhante de flexão $\left(\mathrm{F}_{\mathrm{F}}\right)$, Figura 6 (a), na qual a aba é deformada. Depois de uma determinada carga, existe uma solicitação mecânica na região de solda, que embora não seja uniaxial, tem relação com as propriedades elasto-plásticas do cordão de solda.

Esta relação de cargas é diferente no caso da solda a laser, Figura 5 (c), pois já existe uma tração em junta tripla, portanto, há uma solicitação mecânica uniaxial no reforçador, como pode ser visto na Figura $6(\mathrm{~b})$, onde as forças $\left(\mathrm{F}_{\mathrm{R}}\right)$ aplicadas ao revestimento são iguais.

A partir da Figura 6 pode-se também observar que, depois de uma determinada carga, a força é concentrada na junta. Sendo assim, a capacidade de suportar carga da junta é de fato testada. Portanto, o ensaio de tração T-Pull revelou que o processo de soldagem a laser resiste a uma maior carga na presente configuração, mesmo utilizando chapas de AA6013-T4. Isso se
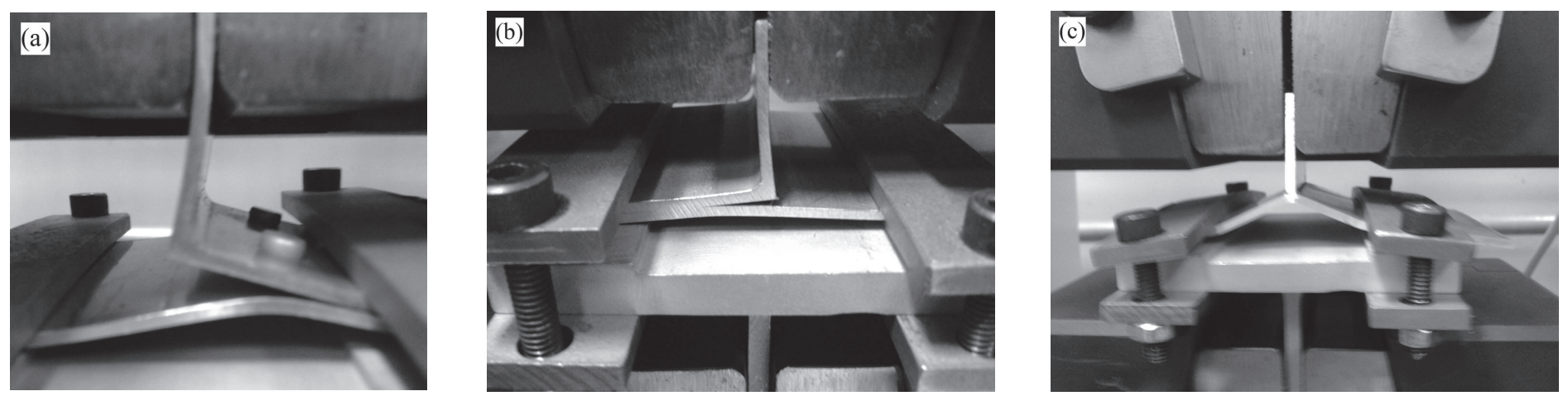

Figura 5. Imagens dos corpos-de-prova durante ensaio de tração T-Pull: (a) rebitado, (b) FSW e (c) LBW.
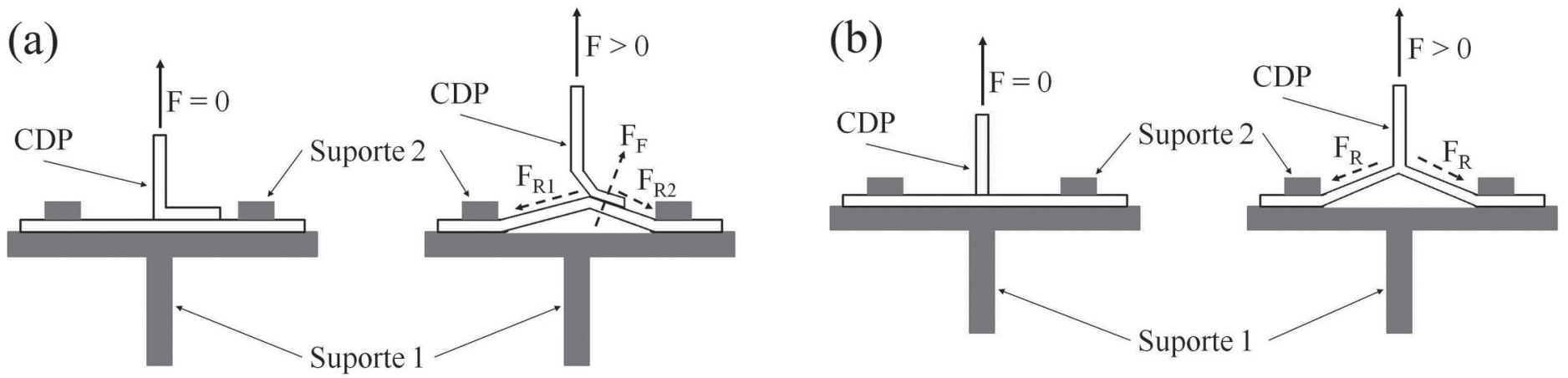

Figura 6. Diagrama de forças presentes durante o ensaio de tração T-Pull: (a) rebitado e soldado por FSW e (b) soldado a laser. 

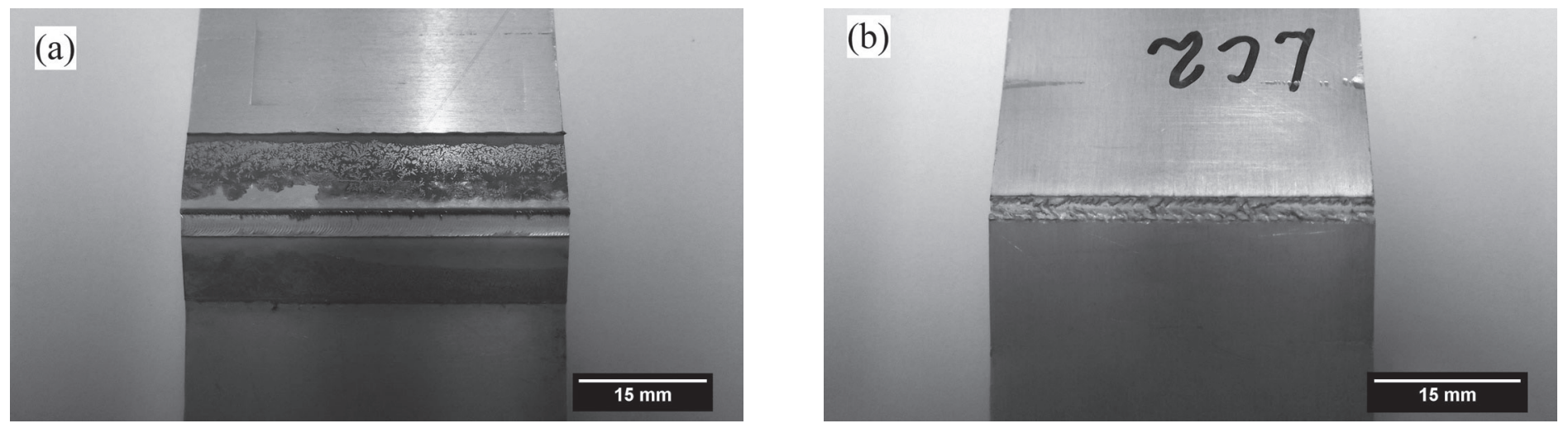

Figura 7. Macrografias da superfície de fratura dos CDPs: (a) FSW e (b) LBW.
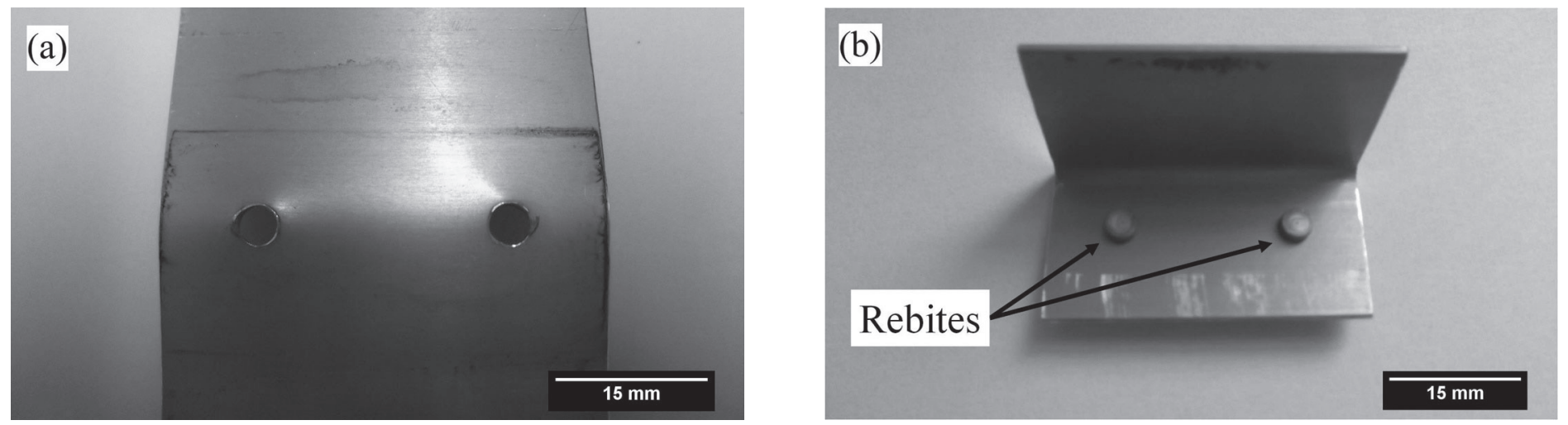

Figura 8. Imagens do corpo-de-prova rebitado após o ensaio de tração T-Pull: (a) revestimento e (b) reforçador.

deve, também, ao fato que as áreas das soldas no caso do FSW e LBW serem maiores que a soma das áreas dos rebites. Isso significa que os processos de soldagem, suportam uma carga maior por efeito de distribuição em uma área maior.

Na Figura 7, são apresentadas as macrografias, da fratura, dos revestimentos dos CDPs soldados por FSW, Figura 7 (a), e a laser, Figura 7 (b), submetidos a ensaio de tração T-Pull. Pode ser visto nas imagens da Figura 7 que os reforçadores dos CDPs soldados por friction stir welding e a laser ao serem solicitados, rompem no cordão de solda e embora exista uma deformação plástica devido ao carregamento, não há trincas macroscópicas aparentes que comprometam o revestimento.

$\mathrm{Na}$ Figura 8 são apresentadas imagens do conjunto revestimento, Figura 8 (a), e reforçador, Figura 8 (b), do corpode-prova rebitado após o ensaio de tração T-Pull. Observa-se que quando o reforçador do corpo-de-prova rebitado é solicitado mecanicamente, os rebites que fazem a conexão do conjunto são arrancados do revestimento, ficando então presos ao reforçador. $\mathrm{O}$ modo de ruptura ocorreu devido às deformações plásticas, advindas das tensões superiores ao escoamento plástico do material do revestimento que, com o aumento nas dimensões do furo, permitiram que o rebite passasse pela abertura.

Na Figura 9, são apresentados os gráficos tensão versus deformação para os ensaios de tração Hoop e na Tabela 4 são apresentados os valores de tensão e deformação máximos. Observa-se que a amostra soldada a laser apresenta uma tensão máxima de $276 \mathrm{MPa}$, sendo $16 \%$ inferior à tensão máxima da amostra rebitada. As amostras soldadas por FSW revelaram uma tensão máxima de $406 \mathrm{MPa}$, sendo $23 \%$ superior à tensão máxima da amostra rebitada. Este valor mais elevado de tensão máxima pode ser justificado pela maior resistência mecânica da liga de alumínio AA 2024 (Ver Tabela 2).

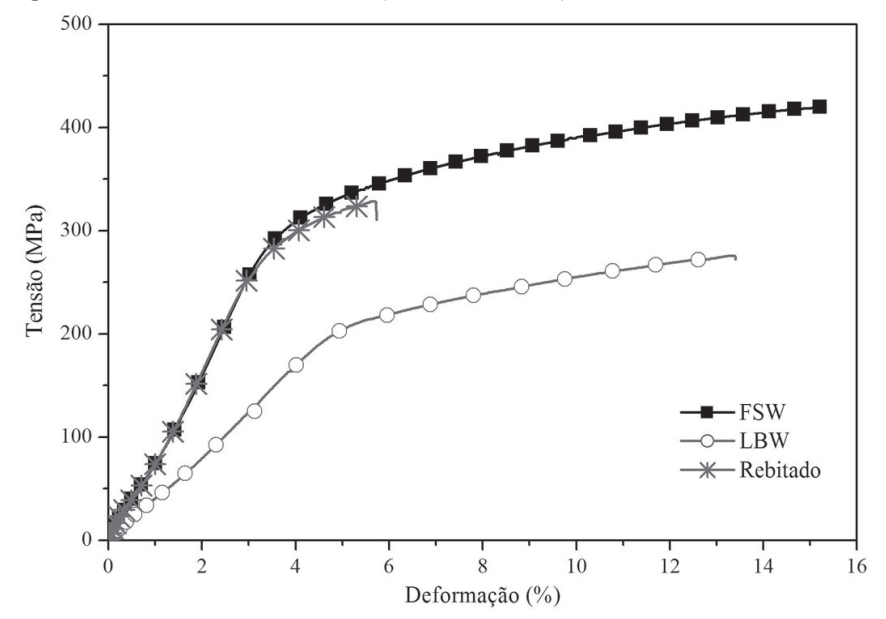

Figura 9. Gráfico de tensão versus deformação para os três corpos-de-prova considerados.

Já a deformação, tanto para as amostras soldadas a laser quanto para as soldadas por friction stir welding apresentaram um aumento de $260 \%$ em relação à junta rebitada. A queda no alongamento nos CDPs rebitados pode estar relacionada com os furos exigidos pelo processo que podem atuar como iniciadores de trincas. 
Tabela 4. Valores das tensões e das deformações máximos para as juntas no ensaio de tração Hoop.

\begin{tabular}{|l|c|c|}
\hline Técnica & Tensão Máxima (MPa) & $\begin{array}{c}\text { Deformação } \\
\text { Máxima (\%) }\end{array}$ \\
\hline FSW & $410 \pm 20$ & $13 \pm 3$ \\
\hline LBW & $276 \pm 1$ & $13 \pm 1$ \\
\hline Rebitado & $329 \pm 1$ & $5 \pm 1$ \\
\hline
\end{tabular}

Na Figura 10, é apresentado o mesmo gráfico da Figura 2, mas incluindo agora para comparação, os valores de tensão máxima, apresentados na Tabela 4. Considerando as ligas do revestimento listadas na Tabela 1, observa-se que os processos de soldagem a laser e por friction stir welding não promovem a queda da resistência mecânica abaixo dos limites de escoamento determinados para as ligas do revestimento utilizadas nestes processos. Já o processo de rebitagem faz com que a resistência mecânica atinja o limite mínimo para a liga de alumínio AA 2024. A ductilidade, com exceção do processo FSW, foi bastante afetada, mostrando uma perda de capacidade de deformação plástica.

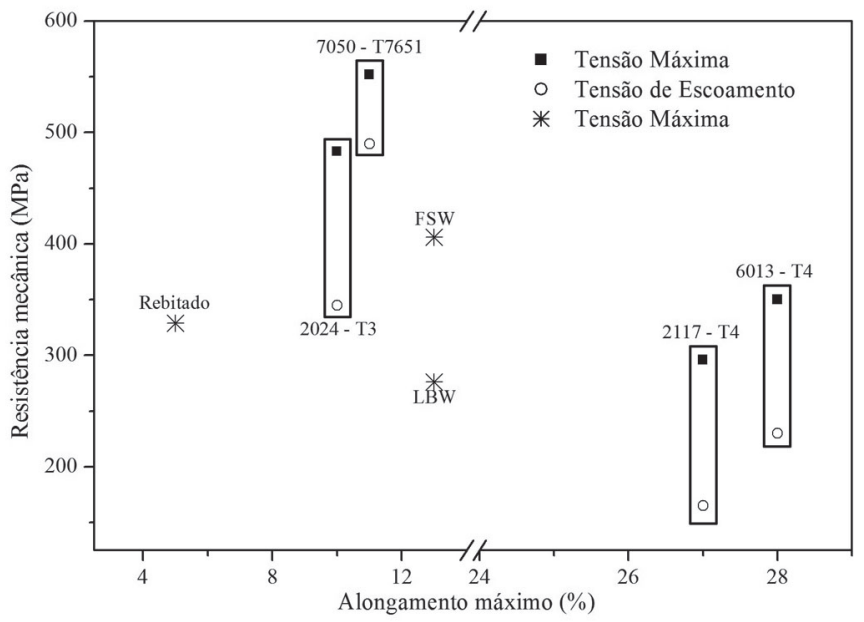

Figura 10. Gráfico da resistência mecânica versus alongamento máximo.

Cada processo de fabricação causa modificações nas propriedades dos materiais. Observa-se na Figura 10, que a tensão máxima está abaixo do limite de escoamento para a liga AA 2024, em decorrência do processo de rebitagem, com exceção dos CDPs soldados pelo processo FSW.

Já o valor de tensão máxima para os CDPs soldados a laser é inferior aos rebitados ou soldados por FSW devido ao fato das propriedades em tração da liga de alumínio AA 6013 serem inferiores ao alumínio classe AA 2024.

Na Figura 11, são apresentadas as macrografias da superfície de fratura dos revestimentos dos CDPs rebitados, Figura 11 (a), soldados por friction stir welding, Figura 11 (b), e soldados a laser, Figura 11 (c), submetidos ao ensaio de tração Hoop.

Na Figura 11 (a), onde é apresentada a macrografia da fratura do CDP rebitado, observam-se superfícies com duas direções diferentes, que se encontram no centro da superfície de fratura. Acredita-se que a ruptura ocorreu a partir dos furos e estendeu-se
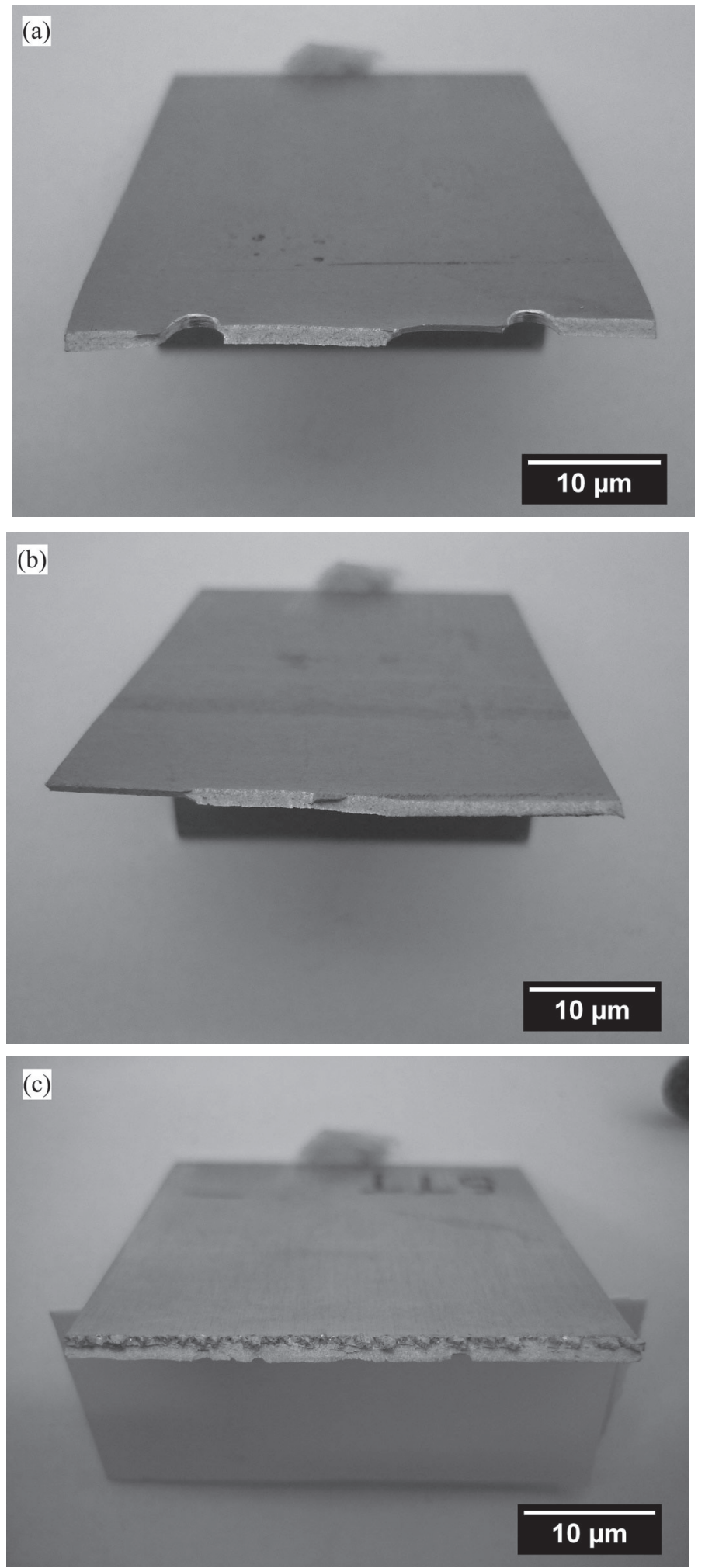

Figura 11. Macrografias da superfície de fratura dos CDPs: (a) Rebitados, (b) FSW e (c) LBW.

até o centro do corpo-de-prova, onde se encontraram e causaram a fratura. Já na Figura 11 (b), observa-se a superfície de fratura do CDP unido por FSW, onde são vistos dois planos de fratura. Neste caso, provavelmente, as trincas se originaram nas laterais e estenderam-se em direção ao centro do CDP. Na Figura 11 
(c), é apresentada a superfície de fratura do corpo-de-prova soldado a laser. Diferentemente das superfícies de fratura dos CDPs rebitados e unidos por FSW, observa-se um único plano de fratura.

\section{Conclusões}

Os resultados das propriedades mecânicas mostraram que há viabilidade técnica para a substituição da rebitagem pela soldagem a laser e por FSW das estruturas de alumínio. Quando o esforço é realizado no sentido do reforçador (stringer), a soldagem a laser apresenta os maiores valores de carga máxima à ruptura. Uma desvantagem do processo de rebitagem é que, quando a solicitação mecânica se faz na forma $T$-Pull, furos são gerados no revestimento, comprometendo a integridade da estrutura. No caso de ensaios Hoop, o qual é mais sensível às propriedades do material de base, as soldas por FSW se mostraram mais tenazes. A solda a laser também se mostrou com boa tenacidade e os CDPs rebitados foram os que apresentaram os piores resultados. A ruptura no caso do FSW ocorreu longe da região da solda, enquanto na solda a laser o material rompeu próximo ao cordão. As superfícies de fratura nas juntas rebitadas indicam que os furos são concentradores de tensão e local de nucleação de trincas.

\section{Agradecimentos}

Os autores agradecem à Empresa Brasileira de Aeronáutica pela doação de material de estudo, à FINEP pelo financiamento do laser e à CAPES por bolsa de estudo de doutoramento.

\section{Referências bibliográficas}

[1] DITTRICH, D. et al., Laser Beam Welding of Hard to Weld Al Alloys for Regional Aircraft Fuselage Design - Frist Results. Physics Procedia, v. 12, p. 113 - 122, abr. 2011.

[2] MENDEZ, P. F.; EAGAR, T. W. Welding processes for aeronautics. Advanced Materials \& Processes, v. 23, p. 39 - 43, maio 2001.

[3] BADINI, C. et al., Laser beam welding of dissimilar aluminium alloys of 2000 and 7000 series: effect of postwelding thermal treatments on $\mathrm{T}$ joint strength. Science and Technology of Welding and Joining, v. 1, n. 6, p. $484-492$.

[4] RÖTZER, I. Laser beam welding makes aircraft lighter. Fraunhoffer Magazine, v. 1, n. 1, p. 36, 2005.

[5] FRANK, P. Laser Beam Welding en Skin-Stringer Joints: Current and New and Al-Alloys. In: European Workshop on Short Distance Welding Concepts for Airframes, 2007, Hamburg, 2007

[6] OLIVEIRA, A. C. Soldagem de alumínio estrutural aeronáutico utilizando um laser a fibra de alta potência. 2011. 151p. Tese (Doutorado) - Instituto Tecnológico de Aeronáutica, São José dos Campos.

[7] SIQUEIRA, R. H. M. Caracterização mecânica e microestrutural de juntas de alumínio $6013 \mathrm{~T} 4$ soldados a laser. 2012. 126p. Dissertação (Mestrado) - Universidade Estadual Paulista, Guaratinguetá.
[8] PRISCO, A. et al., LBW of similar and dissimilar skinstringer joints part I: process optimization and mechanical. Advanced Materials Research, v. 38, 306-319, 2008.

[9] MATWEB - MATERIAL PROPERTY DATA. Disponível em: < http://www.matweb.com>. Acesso em: 06 dez. 2011.

[10] METALS HANDBOOK, Heat Treating, American Society for Metals, Materials Park, Oh. Vol. 4, 8a. Edição, 1973. 